TRANSACTIONS OF THE

AMERICAN MATHEMATICAL SOCIETY

Volume 353, Number 4, Pages 1489-1505

S 0002-9947(00)02724-0

Article electronically published on December 18, 2000

\title{
A CHARACTERISTIC FREE APPROACH TO BRAUER ALGEBRAS
}

\author{
STEFFEN KÖNIG AND CHANGCHANG XI
}

\begin{abstract}
Brauer algebras arise in representation theory of orthogonal or symplectic groups. These algebras are shown to be iterated inflations of group algebras of symmetric groups. In particular, they are cellular (as had been shown before by Graham and Lehrer). This gives some information about block decomposition of Brauer algebras.
\end{abstract}

\section{InTROdUCTION}

Schur-Weyl duality relates the representation theory of the infinite group $G L_{n}(k)$ with that of the symmetric group $\Sigma_{r}$ via the mutually centralising actions of the two groups on the space $\left(k^{n}\right)^{\otimes r}$. Brauer defined the algebras which are now called 'Brauer algebras' (or, somewhat incorrectly, 'Brauer centraliser algebras') by an analogous situation where $G L_{n}$ is replaced by either an orthogonal or a symplectic group and the group algebra of the symmetric group is replaced by a Brauer algebra. More precisely, for a fixed integer $r$ and a given base field $k$, a whole family of Brauer algebras $B_{k}(r, \delta)$ is defined, depending on a parameter $\delta \in k$, which has to be specialised to certain integers to cover the situation Brauer was interested in.

More recently Brauer algebras and their generalisations, especially the BMW algebras, have been looked at in the context of quantum groups and low-dimensional topology (see GHJ).

The classical case of $k$ being the complex numbers (or just having characteristic zero) has been clarified to a large extent by results of Brown, Hanlon and Wales, and Wenzl: If $\delta$ is not an integer of small absolute value, then $B_{k}(r, \delta)$ is semisimple and its simple representations are known. In the other case of $\delta$ being 'singular', Wenzl also obtained a good description of the simple modules. Another such description in the semisimple case has been announced by Kerov. The ring structure of $B_{k}(r, \delta)$, however, seems to be still unknown even in the characteristic zero case.

Recently, Graham and Lehrer have shown that over any field $k$, and for any $\delta$, the Brauer algebra $B_{k}(r, \delta)$ is a cellular algebra. In this way they got a parametrization

Received by the editors January 26, 1998 and, in revised form, January 13, 2000.

1991 Mathematics Subject Classification. Primary 16D25, 16G30, 20G0; Secondary 57M25, 81R05.

Key words and phrases. Brauer algebras, orthogonal groups, symplectic groups, cellular algebras.

Both authors have obtained support from the Volkswagen Foundation (Research in Pairs Programme of the Mathematical Research Institute Oberwolfach). S. König also obtained support from Beijing Normal University during his stay in Beijing in May 1997, when most of this paper has been written. C. C. Xi also obtained support from the Young Teacher Foundation of Chinese Educational Committee and from NSF of China (Grant No. 19831070).

(C)2000 American Mathematical Society 
(which in fact does not depend on $k$ ) of the isomorphism classes of simple modules in general. Considering cellular structures thus opens a way for a characteristic free structure theory of Brauer algebras.

The aim of this paper is to work out such a characteristic free approach and to avoid any complicated computation in doing so by using structural properties instead. We start from scratch and do not use any of the results mentioned above. We first prove that Brauer algebras are cellular (our proof is different from and less computational than that of Graham and Lehrer). More precisely we exhibit in this algebra a structure which we call 'iterated inflation'. As a consequence we do get a new proof not only of the result of Graham and Lehrer, but also of some results of Hanlon and Wales.

Moreover we consider block decompositions of Brauer algebras. Assuming that $\delta$ is not singular (but $k$ is arbitrary) we prove in theorem 7.3 that the Brauer algebra is Morita equivalent to a direct product of group algebras of symmetric groups (which correspond to the layers in the iterated inflation). Hence this case is equivalent to the type $A$ case. As a corollary (7.5) we classify under this assumption the blocks of finite representation type of Brauer algebras - these turn out to be Brauer tree algebras.

We organize the paper as follows: In section two we recall the definition of Brauer algebras and the axiomatics of cellular algebras. In sections three and four we consider inflations, which in general are algebras without unit. They occur as pieces of the iterated inflation as which we write the Brauer algebra in section five. More precisely theorem [5.6 tells us that any Brauer algebra can be written as iterated inflation of certain group algebras of symmetric groups (which are explicitly given). This implies the results of Graham and Lehrer. In section seven we consider Brauer algebras over a field with nonsingular parameter. In this case, we determine the blocks of the Brauer algebra, and among them those of finite representation type.

\section{Definitions}

In this section we recall the definition of Brauer algebras and also that of a cellular algebra, which is our main tool.

2.1. Definition of Brauer algebras. Let $k$ be any field and $n$ a natural number. Let $V$ be the vector space $k^{n}$ on which the group $G L_{n}(k)$ acts naturally, say on the left. Then $G l_{n}(k)$ also acts (diagonally) on the $r$-fold tensor product $V^{\otimes r}$ for any natural number $r$. On this space, also the symmetric group $\Sigma_{r}$ acts on the right, by place permutations. The two actions centralise each other. In particular, the endomorphism ring $\operatorname{End}_{k G l_{n}(k)}\left(V^{\otimes r}\right)$ is a quotient of the group algebra $k \Sigma_{r}$. This setup is called Schur-Weyl duality. Richard Brauer's starting point for defining 'Brauer algebras' was the following question: Which algebra shall replace $k \Sigma_{r}$ in this setup if we replace $G L_{n}(k)$ by either its orthogonal or its symplectic subgroup (in the latter case, of course, $n$ has to be even)? He defined such an algebra by generators and relations, and he also gave an equivalent definition via diagrams. This definition is a special case of the following one, where the parameter $\delta$ has to be chosen as a positive or negative integer (for orthogonal or symplectic groups, respectively).

Definition 2.1. Fix a commutative noetherian domain $k$, an element $\delta \in k$ and a natural number $r$. Then the Brauer algebra $\mathbf{B}_{k}(r, \delta)$ is a $k$-vector space having a 
basis consisting of diagrams of the following form: a diagram contains $2 n$ vertices, $n$ of them called 'top vertices' and the other $n$ called 'bottom vertices', such that the set of vertices is written as a disjoint union of $n$ subsets each of them having two elements; these subsets are called 'edges'. Two diagrams $x$ and $y$ are multiplied by concatenation, that is, the bottom vertices of $x$ are identified with the top vertices of $y$, thus giving rise to edges from the top vertices of $x$ to the bottom vertices of $y$, hence defining a diagram $z$. Then $x \cdot y$ is defined to be $\delta^{m(x, y)} z$, where $m(x, y)$ counts those connected components of the concatenation of $x$ and $y$ which do not appear in $z$, that is, which contain neither a top vertex of $x$ nor a bottom vertex of $y$.

Let us illustrate this definition by an example. We multiply two elements in $B_{k}(4, \delta)$ :

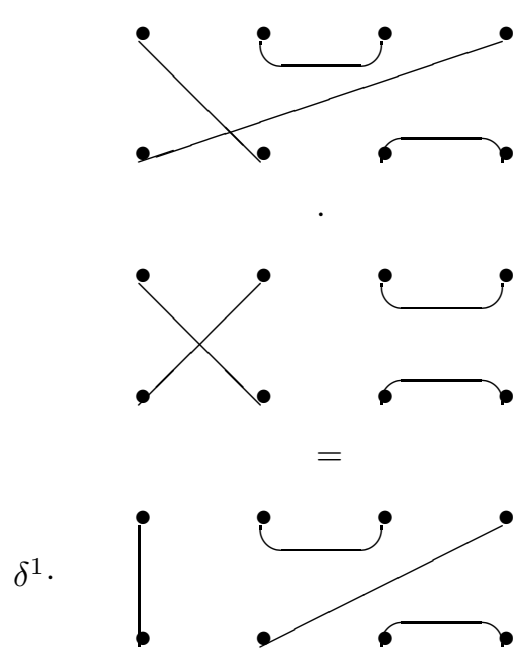

In the literature, the Brauer algebra sometimes is called Brauer centraliser algebra, a term which we will not use, since it is slightly misleading. In fact, in Brauer's original setup, the endomorphism algebra of $V^{\otimes r}$ in general is just a quotient of the Brauer algebra.

2.2. Cellular algebras. First, we recall the original definition of Graham and Lehrer. Then we give our equivalent definition which is convenient to use for looking at the structure of cellular algebras.

Definition 2.2 (Graham and Lehrer, GL]). Let $R$ be a commutative Noetherian integral domain. An associative $R$-algebra $A$ is called a cellular algebra with cell datum $(I, M, C, i)$ if the following conditions are satisfied:

(C1) The finite set $I$ is partially ordered. Associated with each $\lambda \in I$ there is a finite set $M(\lambda)$. The algebra $A$ has an $R$-basis $C_{S, T}^{\lambda}$, where $(S, T)$ runs through all elements of $M(\lambda) \times M(\lambda)$ for all $\lambda \in I$.

(C2) The map $i$ is an $R$-linear anti-automorphism of $A$ with $i^{2}=i d$ which sends $C_{S, T}^{\lambda}$ to $C_{T, S}^{\lambda}$. 
(C3) For each $\lambda \in I$ and $S, T \in M(\lambda)$ and each $a \in A$ the product $a C_{S, T}^{\lambda}$ can be written as $\left(\sum_{U \in M(\lambda)} r_{a}(U, S) C_{U, T}^{\lambda}\right)+r^{\prime}$, where $r^{\prime}$ is a linear combination of basis elements with upper index $\mu$ strictly smaller than $\lambda$, and where the coefficients $r_{a}(U, S) \in R$ do not depend on $T$.

The following is our equivalent definition of cellular algebra:

Definition 2.3 ( $\mathrm{KX} 1]$ ). Let $A$ be an $R$-algebra where $R$ is a commutative Noetherian integral domain. Assume there is an anti-automorphism $i$ on $A$ with $i^{2}=i d$. A two-sided ideal $J$ in $A$ is called a cell ideal if and only if $i(J)=J$ and there exists a left ideal $\Delta \subset J$ such that $\Delta$ is finitely generated and free over $R$ and such that there is an isomorphism of $A$-bimodules $\alpha: J \simeq \Delta \otimes_{R} i(\Delta)$ (where $i(\Delta) \subset J$ is the $i$-image of $\Delta$ ) making the following diagram commutative:

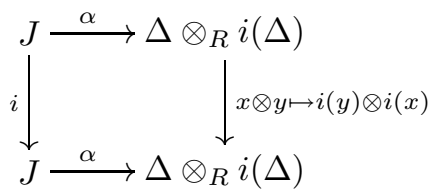

The algebra $A$ (with the involution $i$ ) is called cellular if and only if there is an $R$-module decomposition $A=J_{1}^{\prime} \oplus J_{2}^{\prime} \oplus \cdots \oplus J_{n}^{\prime}$ (for some $n$ ) with $i\left(J_{j}^{\prime}\right)=J_{j}^{\prime}$ for each $j$ and such that setting $J_{j}=\bigoplus_{l=1}^{j} J_{l}^{\prime}$ gives a chain of two-sided ideals of $A: 0=J_{0} \subset J_{1} \subset J_{2} \subset \cdots \subset J_{n}=A$ (each of them fixed by $i$ ) and for each $j(j=1, \ldots, n)$ the quotient $J_{j}^{\prime}=J_{j} / J_{j-1}$ is a cell ideal (with respect to the involution induced by $i$ on the quotient) of $A / J_{j-1}$.

In the following, an $R$-linear anti-automorphism $i$ of $A$ with $i^{2}=i d$ will be called an involution.

Typical examples of cellular algebras are the group algebras of symmetric groups (or more generally, their Hecke algebras) and the classical Schur algebras (whose representation theory gives the polynomial representation theory of $G L_{n}(k)$ over an infinite field $k$ ).

\section{INFLATIONS}

In order to recognise Brauer algebras as cellular algebras and also in order to study block decompositions of Brauer algebras, we will exhibit them as iterated inflations of group algebras of symmetric groups. We have introduced this concept for general cellular algebras in [KX2] and shown (theorem 4.1 in [KX2]) that actually an algebra is cellular if and only if it is such an iterated inflation. Special cases of inflations can be found in the papers of Graham and Lehrer GL, Hanlon and Wales [HW1] and even in the early studies of Brown [Brow1, Brow2, Brow3], who considered 'generalised matrix algebras' which coincide in his case with our inflations.

We first recall the definition of inflations and then look at the structure of inflated algebras. This information is basic for all that follows. Thus we give the details although most of the arguments can be found in several of the above references.

In the following, all tensor products are over the base field $k$ which we omit in the notation. 
3.1. Definitions. Given a $k$-algebra $B$, a $k$-vector space $V$, and a bilinear form $\varphi: V \otimes V \rightarrow B$ with values in $B$, we define an associative algebra (possibly without unit) $A=A(B, V, \varphi)$ as follows: As a $k$-vector space, $A$ equals $V \otimes V \otimes B$. The multiplication is defined on basis elements as follows:

$$
(a \otimes b \otimes x) \cdot(c \otimes d \otimes y):=a \otimes d \otimes x \varphi(b, c) y .
$$

We need an additional property, namely an involution on $A$ : Assume, there is an involution $i$ on $B$. Assume moreover, that $\varphi$ satisfies $i(\varphi(v, w))=\varphi(w, v)$. Then we can define an involution $j$ on $A$ by putting $j(a \otimes b \otimes x)=b \otimes a \otimes i(x)$.

This definition makes $A$ into an associative $R$-algebra (possibly without unit), and $j$ is an involutory anti-automorphism of $A$.

We call $A$ an inflation of $B$ along $V$.

If $V$ has dimension one and the image of $\varphi$ contains the unit element of $B$, then $A$ is clearly isomorphic to $B$. Otherwise, $A$ need not have a unit element, but it may contain idempotents.

Now we are going to produce iterated inflations.

Assume we are given an algebra $B$ (maybe without unit) - which in practise will be an inflation of some other algebra - and an algebra $C$ (with unit). We define an algebra structure on $A:=B \oplus C$ which extends the given structures and which makes $B$ into a two-sided ideal such that $A / B$ becomes isomorphic to $C$. Multiplication is defined by fixing the eight summands of a multiplication map $(B \oplus C) \otimes(B \oplus C) \rightarrow(B \oplus C)$. In order to make $B$ into an ideal we put the summands $B \otimes B \rightarrow C, C \otimes B \rightarrow C$ and $B \otimes C \rightarrow C$ all zero. The summands $C \otimes C \rightarrow C$ and $B \otimes B \rightarrow B$ are defined to be the given multiplications on $C$ and $B$, respectively. Thus we have to choose three bilinear maps $\alpha: C \otimes C \rightarrow B$, $\beta: B \otimes C \rightarrow B$ and $\gamma: C \otimes B \rightarrow B$. Then multiplication in $A$ is defined by $\left(b_{1}+c_{1}\right)\left(b_{2}+c_{2}\right)=b_{1} b_{2}+\beta\left(b_{1}, c_{2}\right)+\gamma\left(c_{1}, b_{2}\right)+\alpha\left(c_{1}, c_{2}\right)+c_{1} c_{2}$.

This multiplication is associative if and only the following conditions are satisfied:

(I) $\beta$ is a homomorphism of left $B$-modules,

(II) $\gamma$ is a homomorphism of right $B$-modules,

(III) for all $b$ in $B$ and $c_{1}, c_{2}$ in $C$ there is an equality: $\beta\left(\beta\left(b, c_{1}\right), c_{2}\right)=b \alpha\left(c_{1}, c_{2}\right)+$ $\beta\left(b, c_{1} c_{2}\right)$,

(IV) for all $b$ in $B$ and $c_{1}, c_{2}$ in $C$ there is an equality: $\gamma\left(c_{1}, \gamma\left(c_{2}, b\right)\right)=\gamma\left(c_{1} c_{2}, b\right)+$ $\alpha\left(c_{1}, c_{2}\right) b$,

(V) for all $c_{1}, c_{2}, c_{3}$ in $C$ there is an equality: $\alpha\left(c_{1} c_{2}, c_{3}\right)+\beta\left(\alpha\left(c_{1}, c_{2}\right), c_{3}\right)=$ $\alpha\left(c_{1}, c_{2} c_{3}\right)+\gamma\left(c_{1}, \alpha\left(c_{2}, c_{3}\right)\right)$,

(VI) for all $b_{1}, b_{2}$ in $B$ and $c$ in $C$ there is an equality: $\beta\left(b_{1}, c\right) b_{2}=b_{1} \gamma\left(c, b_{2}\right)$,

(VII) for all $c_{1}, c_{2}$ in $C$ and $b$ in $B$ there is an equality: $\beta\left(\gamma\left(c_{1}, b_{1}\right), c_{2}\right)=$ $\gamma\left(c_{1}, \beta\left(b, c_{2}\right)\right)$.

We need another condition in order to make sure that $A$ has a unit element which is mapped to the unit element $1(C)$ of $C$ by the quotient homomorphism.

(U) There exists an element $b$ in $B$ such that $b+1(C)$ is a unit element in $A$.

Some other (obvious) conditions are needed in order to extend the given involutions on $B$ and $C$ to an involution on $A$. More conditions (again all of them obvious) make sure that all the inflation pieces are subquotients of ideals in the algebra which is the result of the iterated inflation.

Applying this procedure inductively to algebras $C, B_{1}, B_{2}, \ldots, B_{n}$ where each $B_{j}$ itself is an inflation of an algebra $B_{j}^{\prime}$, we define an iterated inflation $A$ of $C_{1}, B_{1}^{\prime}, B_{2}^{\prime}, \ldots, B_{n}^{\prime}$. 
In KX2] (theorem 4.1) we have shown that an algebra is cellular if and only if it can be written as an iterated inflation of copies of full matrix algebras. Moreover ([KX2], proposition 3.4) an iterated inflation of cellular algebras always is cellular again.

3.2. Inflated algebras. Let $B$ be any algebra, $\varphi: V \otimes V \rightarrow B$ a bilinear form (for any finite dimensional $k$-vector space $V$ ) and $A=V \otimes V \otimes B$ the resulting inflation of $B$. We are going to discuss the ring structure of $A$.

Since $B$ need not have a unit element, we have to be careful, when talking about its radical. By radical we always mean the Jacobson radical which is the intersection of the annihilators of the simple left modules and which contains any nilpotent ideal.

The following lemma is obvious.

Lemma 3.1. (a) The vector space $I:=V \otimes V \otimes \operatorname{rad}(B)$ is a two-sided ideal of $A$ which is contained in $\operatorname{rad}(A)$. Moreover, $I$ is nilpotent of nilpotence degree smaller than or equal to the nilpotence degree of $B$.

(b) If $B$ admits a decomposition $B=B_{1} \oplus B_{2}$ into two-sided ideals, then $A$ also admits a decomposition $A=\left(V \otimes V \otimes B_{1}\right) \oplus\left(V \otimes V \otimes B_{2}\right)$.

For all the questions we will be interested in later on, we may without loss of generality assume that $B$ has the following properties:

(1) It is semisimple, since by corollary 8.4 in $\mathrm{KX} 2$ the maximal semisimple quotient of a cellular algebra carries an induced cellular structure.

(2) It is indecomposable as a ring, since a block of a cellular algebra again is cellular.

Hence it is simple and thus (because it is cellular, see [KX1], proposition 3.4) a full matrix ring over $k$.

We decompose $B$ as a left and as a right module into ${ }_{B} B=\bigoplus L_{m}$ and $B_{B}=$ $\bigoplus R_{m}$. Finally, we fix a basis $v_{1}, \ldots, v_{l}$ of $V$.

Lemma 3.2. Let $V$ be a non-zero vector space. Fix a basis $v_{1}, \ldots, v_{l}$ of $V$ and $a$ decomposition of the left $B$-module $B$ into simple submodules as follows: ${ }_{B} B=$ $\bigoplus_{m} L_{m}$.

(a) For each $m$ and $n$ the vector space $V \otimes v_{n} \otimes L_{m}$ is a left $A$-module which we denote by $P(m, n)$.

(b) Fix $m$ and $n$. Denote by $N(m, n)$ the subspace of $P(m, n)$ defined as follows: An element $\sum_{v \in V, l \in L_{m}} v \otimes v_{n} \otimes l$ is in $N(m, n)$ if and only if for all $w \in V$, the $\operatorname{sum} \sum_{v, l} \varphi(w, v) l$ equals zero.

Then $N(m, n)$ is a submodule of $P(m, n)$, and every element of $A$ acts as zero (from the left) on $N(m, n)$.

(c) If $\varphi \neq 0$, then the quotient module $P(m, n) / N(m, n)$ is simple. Denote it by $L(m, n)$.

(d) If $\varphi=0$, then the multiplication in $A$ is zero.

Proof. (a) By definition, a basis element $u \otimes v \otimes b$ of $A$ acts on an element $w \otimes v_{m} \otimes x$ on the left by sending it to $u \otimes v_{m} \otimes b \varphi(v, w) x$, which is an element of $P(m, n)$. Thus $P(m, n)$ is a submodule of $A$. As a vector space, $A$ is a direct sum of the subspaces $V \otimes v_{m} \otimes L_{n}$ (where $m$ and $n$ run), hence each of them is a module. This proves (a). 
(b) Let $u \otimes w \otimes x$ be an element in $A$ and multiply it with an element of $N(m, n)$. We get

$$
(u \otimes w \otimes x) \cdot\left(\sum_{v \in V, l \in L_{m}} v \otimes v_{n} \otimes l\right)=u \otimes v_{n} \otimes x\left(\sum_{v, l} \varphi(w, v) l\right)=0 .
$$

(c) If $\varphi \neq 0$, then $N(m, n) \neq P(m, n)$. In fact, if $P(m, n)=N(m, n)$, then for any $w, v \in V$ and $l \in L_{m}$ we have $\varphi(w, v) l=0$. This implies that $\varphi(w, v) L_{m}=0$. Since $B$ is a simple artinian algebra, $L_{m}$ is a faithful $B$-module. Thus $\varphi(w, v)=0$ for all $w, v \in V$, which is a contradiction. Hence $N(m, n)$ is a proper subspace of $P(m, n)$.

Pick an element $z:=\sum_{v \in V, x \in L_{m}} v \otimes v_{n} \otimes x$ which is in $P(m, n)$, but not in $N(m, n)$. We show that $z$ generates all of $P(m, n)$ as a left $A$-module. Multiplying $z$ with an element of $A$ from the left, we get the following expression:

$$
(u \otimes w \otimes x) \cdot\left(\sum_{v \in V, l \in L_{m}} v \otimes v_{n} \otimes l\right)=u \otimes v_{n} \otimes x\left(\sum_{v, l} \varphi(w, v) l\right),
$$

in which $u, w$, and $x$ can be chosen arbitrarily. Since $z$ is not in $N(m, n)$ we can choose $w$ in such a way that $\sum_{v, l} \varphi(w, v) l$ is not zero. But then we can let $x$ vary in such a way that the expression $x\left(\sum_{v, l} \varphi(w, v) l\right)$ runs through all of $L_{m}$ (which is simple). Hence the claim follows.

This implies that any non-zero element in $P(m, n) / N(m, n)$ generates all of it, hence this is a simple $A$-module.

(d) This is trivial by the definition of $A$.

We note that similar statements hold true for right modules. In order to formulate them appropriately for later use, one has to observe that the involution $i$ on $A$ restricts to a $k$-isomorphism $V \rightarrow V$ which identifies the first and the second copy of $V$ in $V \otimes V \otimes B=A$. This provides $V$ with another basis $i\left(v_{1}\right), i\left(v_{2}\right), \ldots, i\left(v_{l}\right)$.

We note that both the description of the maximal semisimple quotient of $A$ immediately carries over to the general situation where $B$ is not assumed to be semisimple.

We have to determine the radical of the algebra $A$. The previous lemma gives us an obvious candidate, since it provides us with simple modules of the form $P(m, n) / N(m, n)$. We shall show that there really is a simple quotient algebra having as simple left modules precisely the $L(m, n)$.

Lemma 3.3. Let $\hat{N}_{L}:=\sum_{m, n} N(m, n)$, and $\hat{N}_{R}$ the analogous sum of right modules. Let $\hat{N}$ be the sum (of vector spaces)

$$
\hat{N}:=\hat{N}_{L}+\hat{N}_{L} \cdot A+\hat{N}_{R}+A \cdot \hat{N}_{R},
$$

where $\hat{N}_{L} \cdot A$ is the $k$-space generated by products of the form $n \cdot a$ with $a \in A$ and $n \in \hat{N}_{L}$. Then $\hat{N}$ is a two-sided ideal of $A$, it is nilpotent of degree three, i.e. $\hat{N}^{3}=0$, and $A / \hat{N}$ is a simple algebra which is Morita equivalent to $\operatorname{End}_{k}(L(m, n))$ for any $m, n$. In particular, $\hat{N}$ equals the Jacobson radical of $A$.

The proof of this lemma will be postponed to the end of the next section. Let us first state a few corollaries of the lemma.

Corollary 3.4. If $\hat{N}$ is not equal to $A$, then $A / \hat{N}$ is Morita equivalent to $B$.

Corollary 3.5. The algebra $A$ has a unit element if and only if $N(m, n)=0$ for all $m, n$, or equivalently, $N(m, n)=0$ for some $m$ and $n$. 
Proof. If $A$ has a unit, say $e$, then $e$ must act non-trivially on $\hat{N}$, which implies that $N(m, n)$ equals zero for all $m, n$. Conversely, if $N(m, n)$ is zero, then $A$ is a simple artinian ring, hence has a unit element.

We now have precise knowledge on the simple $A$-modules. To be consistent with our use of (Jacobson) radical, a module $M$ has to be called simple if $A M \neq 0$ and there are no non-trivial submodules. Hence we have the following description of simple modules.

If $\hat{N}=0$, then $A$ has precisely one isomorphism class of simple modules, represented by any indecomposable direct summand of $A$. Moreover, $A$ acts nontriviallly on any non-zero $A$-module.

If $0 \neq \hat{N} \neq A$, then $A$ has precisely one isomorphism class of simple modules, again represented by any indecomposable direct summand of $A / \hat{N}$. But there is also the module $k$ with trivial $A$-action.

If $A=\hat{N}$, then $A$ has no simple module, but there is again the module $k$ with trivial $A$-action.

Suppose $B$ is semisimple. We call the bilinear form $\varphi$ singular if $N_{\varphi}(L, n) \neq$ 0 for some simple $B$-module $L$ and $n$, where $N_{\varphi}(L, n)$ stands for the subset of $V \otimes v_{n} \otimes L$ consisting of all elements $\sum_{v \in V, l \in L} v \otimes v_{n} \otimes l$ with $\sum_{v, l} \varphi(w, v) l=0$ for all $w \in V$.

If $B$ is arbitrary, we call $\varphi$ singular if $\bar{\varphi}: V \otimes V \longrightarrow B \longrightarrow B / \operatorname{rad}(B)$ is singular.

\section{Matrix PRESEntation OF INFLAted ALGEBRAS}

In this section we use matrices to present and study the inflated algebras (see Brown's generalised matrix algebras Brow1). We assume that $V$ is a non-zero vector space with a basis $\left\{v_{1}, \cdots, v_{m}\right\}$ and that $B$ is an arbitrary $k$-algebra with identity. Again we denote by $A$ the inflated algebra $(B, V, \varphi)$. Let $M_{m}(B)$ denote the full matrix algebra over $B$.

Clearly, the bilinear form $\varphi$ can be described by an $m \times m$ matrix $\Phi$ over $B$, that is, $\Phi=(\varphi(i, j))$, where $\varphi(i, j)=\varphi\left(v_{i}, v_{j}\right)$ for all $1 \leq i, j \leq m$. Now we use this matrix to define a new multiplication $\circ$ on $M_{m}(B)$ in the following way:

$$
X \circ Y:=X \Phi Y \text { for all } X, Y \in M_{m}(B) \text {. }
$$

This multiplication is associative and hence $\left(M_{m}(B), \circ\right)$ is an associative algebra. (The addition of two matrices is the usual matrix addition). We denote this algebra by $\left(M_{m}(B), \Phi\right)$.

The following lemma shows that the inflated algebra $A$ can be described by this new algebra.

Lemma 4.1. The inflated algebra $A(B, V, \varphi)$ is isomorphic to $\left(M_{m}(B), \Phi\right)$ as $k-$ algebra.

Proof. Since each element of $V \otimes V \otimes B$ can be uniquely written in the form

$$
\sum_{i, j}^{m} v_{i} \otimes v_{j} \otimes b_{i j}
$$

this gives us a matrix $\left(b_{i j}\right) \in M_{m}(B)$. Thus we may define a linear map (depending on the choice of basis in $V$ )

$$
\mu: A=V \otimes V \otimes B \longrightarrow\left(M_{m}(B), \Phi\right), \quad \sum_{i, j} v_{i} \otimes v_{j} \otimes b_{i j} \mapsto\left(b_{i j}\right) .
$$


It is easy to check that this is an algebra homomorphism. Comparing the dimensions of the two spaces, we see easily that $\mu$ is an isomorphism.

From this matrix presentation of inflated algebras, we have the following fact. Note that in an artinian algebra left and right invertibility is the same, hence a left unit is a right unit and so on.

Proposition 4.2. The following are equivalent for the inflated algebra $A$ :

(1) The algebra $A$ has a unit element.

(2) The matrix $\Phi$ is invertible, that is, there is a matrix $X \in M_{m}(B)$ such that $\Phi X=I_{m}$, where $I_{m}$ denotes the identity matrix in $M_{m}(B)$.

(3) The matrix $\bar{\Phi}:=(\overline{\varphi(i, j)}) \in M_{m}(\bar{B})$ is invertible, where $\bar{B}$ stands for $B / \operatorname{rad}(B)$ and $\bar{b}$ stands for the residue class of an element $b \in B$.

Proof. The equivalence of (1) and (2) follows from Lemma 4.1. It is obvious that (3) is implied by (2).

Now assume that $\bar{\Phi}$ is invertible, that is, there is a matrix $X=\left(x_{i j}\right) \in M_{m}(B)$ such that $\bar{\Phi} \bar{X}=I_{m} \in M_{m}(\bar{B})$, where $\bar{X}=\left(\bar{x}_{i j}\right) \in M_{m}(\bar{B})$. Since $\operatorname{rad}\left(M_{m}(B)\right)=$ $M_{m}(\operatorname{rad}(B))$ and $M_{m}(B / \operatorname{rad}(B))=M_{m}(B) / \operatorname{rad}\left(M_{m}(B)\right)$, there is a matrix $Z \in$ $M_{m}(\operatorname{rad}(B))$ such that $\Phi X-I_{m}=Z$. Since $Z$ is a nilpotent matrix over $B$, the matrix $I_{m}+Z$ is invertible in $M_{m}(B)$. This shows that the matrix $\Phi X$ is invertible and therefore $\Phi$ is invertible.

Corollary 4.3. If $\Phi$ is invertible in $M_{m}(B)$, then for any $B$-module $M$ we have $N_{\varphi}(M, n)=0$.

Proof. As remarked above, the fact that $M_{m}(B)$ is an artinian algebra implies that always $X \Phi=I_{m}$ if $\Phi X=I_{m}$ holds true.

Suppose $\sum_{i}^{t} u_{i} \otimes v_{n} \otimes l_{i} \in N(M, n)$, where we may assume that $l_{1}, \cdots, l_{t}$ are $k$-linear independent. By definition, we have for any $v_{j}$ that $\sum_{i} \varphi\left(v_{j}, u_{i}\right) l_{i}=0$. Now let $u_{j}=\sum_{k} \alpha_{j k} v_{k}$. Then $\sum_{k=1}^{m} \sum_{i=1}^{t} \alpha_{i k} \varphi\left(v_{j}, v_{k}\right) l_{i}=0$. If $X=\left(x_{i j}\right)$, then multiplying by $x_{s, j}$ and summing up over $j$, we have

$$
0=\sum_{i, k} \alpha_{i k} \sum_{j} x_{s j} \varphi\left(v_{j}, v_{k}\right) l_{i}=\sum_{i, k} \alpha_{i k} \delta_{s k} l_{i}=\sum_{i} \alpha_{i s} l_{i} .
$$

Since $l_{1}, \cdots, l_{t}$ are linear independent, $\alpha_{i s}=0$ for all $i$ and $s$. Hence $u_{i}=0$ for all $i$.

Now let us give the proof of lemma 3.3.

Proof of 3.3. We keep all notation introduced before.

Assume now that $B$ is a matrix algebra $M_{s}(k)$ over $k$. The algebra $A$ acts trivially from the left on $\hat{N}_{L}$ and from the right on $\hat{N}_{R}$. It follows that $\hat{N}$ is a twosided ideal. Products of elements in $\hat{N}$ are elements of $\hat{N}_{L} \cdot \hat{N}_{R}+\hat{N}_{L} \cdot A \cdot \hat{N}_{R}$. But this space is annihilated by all of $A$ both from the left and from the right. Hence triple products of elements in $\hat{N}$ are zero. Thus $\hat{N}$ is contained in the Jacobson radical of $A$.

Define $E\left(\begin{array}{c}i j \\ \lambda \mu\end{array}\right)=v_{i} \otimes v_{j} \otimes E_{\lambda \mu}$, where $E_{\lambda \mu}$ is the matrix units in $M_{s}(k)$. Then the elements $E\left(\begin{array}{c}i j \\ \lambda \mu\end{array}\right)=v_{i} \otimes v_{j} \otimes E_{\lambda \mu}, 1 \leq i, j \leq l, 1 \leq \lambda, \mu \leq s$, form a basis of $A$, and the multiplication in $A$ with respect to this basis reads as follows:

$$
E\left(\begin{array}{c}
i j \\
\lambda \mu
\end{array}\right) E\left(\begin{array}{c}
m n \\
\gamma \delta
\end{array}\right)=\varphi\left(\begin{array}{c}
j m \\
\mu \gamma
\end{array}\right) E\left(\begin{array}{c}
i n \\
\lambda \delta
\end{array}\right)
$$


where $\varphi\left(\begin{array}{c}i j \\ \lambda \mu\end{array}\right)$ is defined by

$$
\varphi\left(v_{i}, v_{j}\right)=\sum_{\lambda, \mu} \varphi\left(\begin{array}{c}
i j \\
\lambda \mu
\end{array}\right) E_{\lambda \mu} .
$$

Define $\Phi=\left(\varphi\left(\begin{array}{c}i j \\ \lambda \mu\end{array}\right)\right)$ to be the matrix with $\left(\left(\begin{array}{l}i \\ \lambda\end{array}\right),\left(\begin{array}{c}j \\ \mu\end{array}\right)\right)$-th entry $\varphi\left(\begin{array}{c}i j \\ \lambda \mu\end{array}\right)$; then by a similar argument as in Brow1 we will prove that the radical of $A$ has the $k$ dimension $l^{2} s^{2}-r^{2}$, where $r$ is the rank of the matrix $\Phi$.

We fix a left ideal $L_{\mu_{0}}$ in $B$ and its basis $\left\{E_{\lambda \mu_{0}}\right\}$. Let $\sigma: V \otimes L_{\mu_{0}} \longrightarrow V \otimes L_{\mu_{0}}$ be the linear map defined by $v_{i} \otimes E_{\lambda \mu_{0}} \mapsto \sum_{j, \mu} \varphi\left(\begin{array}{c}j i \\ \mu \lambda\end{array}\right) v_{j} \otimes E_{\mu \mu_{0}}$. Clearly, the linear map has $\Phi$ as its matrix. The kernel of $\sigma$ consists precisely of the elements of the form $\sum_{i, \lambda} \alpha_{i \lambda} v_{i} \otimes E_{\lambda \mu_{0}}$ with $\sum_{i, \lambda} \varphi\left(\begin{array}{c}j i \\ \mu \lambda\end{array}\right) \alpha_{i \lambda}=0$ for all $j$ and $\mu$. An equivalent condition is that $\sum_{i, \lambda} \alpha_{i \lambda} v_{i} \otimes v_{n} \otimes E_{\lambda \mu_{0}}$ lies in $N\left(\mu_{0}, n\right)$. Now we identify $A$ with $\left(V \otimes L_{\mu_{0}}\right) \otimes\left(V \otimes L_{\mu_{0}}\right)$ via the $k$-linear map $v_{i} \otimes v_{j} \otimes E_{\lambda \mu} \mapsto\left(v_{i} \otimes E_{\lambda \mu_{0}}\right) \otimes\left(v_{j} \otimes E_{\mu \mu_{0}}\right)$. Thus, as in [HW1], we prove that the dimension of $A / \hat{N}$ is $r^{2}$. (Note that the dimension of the image of $\sigma$ is the rank of the matrix $\Phi$.) Hence the dimension of $\hat{N}$ is equal to the dimension of the radical $A$. It follows from $\hat{N} \subset \operatorname{rad}(A)$ that the radical of $A$ coincides with $\hat{N}$. Now the rest of Lemma 3.3 becomes transparent.

Let us note here that lemma 4.1 shows that an inflation is a generalized matrix algebra in the sense of Brown [Brow1. In his language, an equivalent version of the above proof runs as follows: The isomorphism class of the algebra $A$ is not changed if we replace the 'multiplication matrix' $\Phi$ by a matrix $S \Phi T$ (with $S$ and $T$ being invertible). Hence one can replace $\Phi$ by its normal form, with some 1's on the diagonal and 0 's elsewhere, say $\left(\begin{array}{cc}I_{r} & 0 \\ 0 & 0\end{array}\right)$ with $I_{r}$ denoting an identity matrix of size $r \times r$ and 0 denoting matrices of appropriate sizes. Then clearly $\hat{N}_{L}$ consists of matrices of the form $\left(\begin{array}{cc}0 & 0 \\ \star & \star\end{array}\right)$ (with $\star$ denoting arbitrary entries), and $\hat{N}_{R}$ consists of matrices of the form $\left(\begin{array}{cc}0 & \star \\ 0 & \star\end{array}\right)$. The two-sided ideal $\hat{N}$ generated by them according to the multplication rule in $A$ consists of matrices of the form $\left(\begin{array}{cc}0 & \star \\ \star & \star\end{array}\right)$. Hence the quotient algebra $A / \hat{N}$ has a basis represented by matrices of the form $\left(\begin{array}{cc}\star & 0 \\ 0 & 0\end{array}\right)$, which implies the assertion of the lemma.

Under an additional assumption, which is easily seen to be satisfied by Brauer algebras, one can avoid some of the above computations by directly writing down a set of primitive idempotents in $A$. Assume that for the given basis $v_{1}, \ldots, v_{l}$ all elements $\varphi\left(v_{j}, v_{j}\right)$ are non-zero scalar multiples of the identity of $B$. Then elements of the form

$$
\frac{1}{\varphi\left(v_{j}, v_{j}\right)} v_{j} \otimes v_{j} \otimes e
$$

(for $e$ a primitive idempotent in $B$ ) are primitive idempotents.

\section{Brauer ALGEBRAs ARE ITERATED INFLATIONS}

In this section we prove the main technical result of this paper: Brauer algebras are iterated inflations of group algebras of symmetric groups. We observed this fact already in $\mathrm{KX} 2$ - without giving details of the proof - as a consequence of the 
rather complicated computations in [GL]. The proof we give here is much more elementary, and it works for related 'diagram algebras' as well. (We have checked this for Temperley-Lieb algebras and for Jones' annular algebras.)

As an immediate consequence we get that Brauer algebras are cellular, and we also obtain a description of the simple modules of the Brauer algebra over a field.

We fix a commutative noetherian domain $k$, a natural number $r$, a parameter $\delta \in k$, and the corresponding Brauer algebra $A:=B_{k}(r, \delta)$. Note that $k$ need not be a field, so we cover in particular the case of $\delta$ being an indeterminate over a given field. It is well-known how to define an involution on $A$ :

Lemma 5.1. Sending a diagram d to a digram $i(d)$ which has the same edges, but whose top and bottom vertices are interchanged (that is, the vertex $l$ of $d$ is the vertex $r+l$ of $i(d)$ and the vertex $r+l$ of $d$ is the vertex $l$ of $i(d)$ for all $l$ with $1 \leq l \leq r)$, defines an involution $i: A \rightarrow A$.

The first step in exhibiting the structure of an iterated inflation consists in defining an appropriate filtration which also is well-known:

Lemma 5.2. Assign to a diagram $d$ the number $t(d)$ of 'through strings', that is, edges which connect a top vertex with a bottom vertex. Let $J_{l}$ be the $k$-space generated by all diagrams with $t(d) \leq l$. Then $J_{l}$ is a twosided ideal of $A$. This defines a filtration of $A$ by twosided ideals: $0 \subset J_{a} \subset J_{a+2} \subset \cdots \subset J_{r-2} \subset J_{r}$ (which starts with either $a=0$ or $a=1$ depending on whether $r$ is even or odd).

Proof. If a diagram $d$ has $t(d)$ through strings, then it has $r-2 t(d)$ edges which connect top vertices with top vertices and it also has $r-2 t(d)$ edges which only involve bottom vertices. Multiplication of two diagrams $d_{1}$ and $d_{2}$ yields a scalar multiple of a diagram $d_{3}$ with $t\left(d_{3}\right) \leq \min \left\{t\left(d_{1}\right), t\left(d_{2}\right)\right\}$, since those edges of $d_{1}$ involving only top vertices are not changed by the multiplication, and the same holds for the 'bottom' edges of $d_{2}$.

In the following we use the convention $k \Sigma_{0}=k$ in order to simplify notation.

Lemma 5.3. Fix an index $l$ and let $B$ be the $k$-algebra (possibly without unit) $B:=J_{l} / J_{l-2}$. Then $B$ is isomorphic (as a $k$-algebra) to an inflation $V_{l} \otimes V_{l} \otimes k \Sigma_{l}$ of the group algebra of the symmetric group $\Sigma_{l}$ along a free $k$-module $V_{l}$. The $k-$ rank of $V_{l}$ equals the number of possibilities to draw $(r-l) / 2$ edges between $r-l$ out of $r$ vertices. The bilinear form $\varphi$ will be defined in the proof.

We remark that the mentioned number of configurations of $f:=(r-l) / 2$ edges in the top row of a diagram equals

$$
\frac{r !}{2^{f} l ! f !}
$$

hence the dimension of $B$ equals

$$
\frac{(r !)^{2}}{4^{f} l !(f !)^{2}}
$$

Proof. Denote the vector space $V_{l} \otimes V_{l} \otimes k \Sigma_{l}$ by $C$. We define a $k$-isomorphism $\psi: B \rightarrow C$ as follows: $B$ has a $k$-basis consisting of diagrams $d$ with $t(d)=l$, that is, there are $(r-l) / 2$ edges connecting top vertices with top vertices, another $(r-l) / 2$ edges connecting bottom vertices with bottom vertices, and another $l$ through strings. Fix a diagram $d$. Denote by $e$ the configuration formed by the 
edges relating top vertices only and by $f$ the configuration formed by the edges relating bottom vertices only. Renumber the top vertices of the through strings as $1, \ldots, l$ (from left to right) and their bottom vertices also (from left to right) as $1, \ldots, l$. Then the through strings define a permutation $\sigma$ of the set of $l$ letters by sending the numbers assigned to the top vertex of a string to the numbers of its bottom vertex.

Define $\psi: B \rightarrow C$ to send $d$ to

$$
\psi(d):=e \otimes f \otimes \sigma .
$$

This clearly defines an isomorphism of $k$-modules.

In order to give $C$ a multiplicative structure we have to define $\varphi$. This will be done using the isomorphism $\psi$, thus verifying at once that $\psi$ is a ring isomorphism. Let $x:=e \otimes f \otimes \sigma$ and $y:=g \otimes h \otimes \tau$ be two basis elements of $C$. The product $x y$ has to be of the form $e \otimes h \otimes \sigma \varphi(f, g) \tau$. We have to distinguish two cases.

First case: Each edge in $f$ has at least one common vertex with an edge in $g$ (after identifying as usual the vertices in $f$ with those in $g$ and thus forming a graph $\Gamma$ consisting of these vertices together with all edges of $f$ and of $g$ ). Then we define $\varphi(f, g):=\delta^{m} \cdot \sigma$ where the data $m$ and $\sigma$ are defined as follows: The group element $\sigma$ is the permutation defined by the through strings of the product $\psi^{-1}(f \otimes f \otimes I d) \cdot \psi^{-1}(g \otimes g \otimes I d)$. The number $m$ counts the connected components of the graph $\Gamma$, which is defined as follows: Denote the bottom vertices of $e$ by $r+1, r+2, \ldots, 2 r$ and the bottom vertices of $f$ by $1,2, \ldots, r$. Then identify $r+j$ with $j$ for each $j, 1 \leq j \leq r$. The set of edges of $\Gamma$ is the union of the set of edges in $e$ and the set of edges in $f$.

The product $x y$ defined in this way obviously coincides with $\psi\left(\psi^{-1}(x) \psi^{-1}(y)\right)$.

Second case: There is at least one edge $\gamma$ in $f$ which does not share a vertex with any edge in $g$. Then we define $\varphi(f, g):=0$. We have to show that this fits together with multiplication in $B$. In fact, forming the product of the two diagrams representing $\psi^{-1}(x)$ and $\psi^{-1}(y)$ inside $A$ gives a result which has a strictly smaller number of through strings, since the edge $\gamma$ yields an additional bottom edge in the product. Hence, inside $B$ the product is zero.

This defines $\varphi$ and shows that $\psi$ is an isomorphism of $k$-algebras.

It is easy to check that $\psi$ 'preserves' the involution $i$ :

Lemma 5.4. Under $\psi$ the involution $i: B \rightarrow B$ corresponds to the standard involution on $C$ which sends $e \otimes f \otimes \sigma$ to $f \otimes e \otimes \sigma^{-1}$.

This finishes our consideration of the candidate layers of the iterated inflation. It remains to make sure, that the layers fit together (which is more than just having a filtration by two-sided ideals). For simplicity we denote all the isomorphisms identifying a layer with some inflation by the same letter $\psi$.

Lemma 5.5. Let $d_{1} \in J_{m} \backslash J_{m-2}$ and $d_{2} \in J_{n} \backslash J_{n-2}$ be two diagrams in $A$ and let their respective $\psi$-images be $a \otimes b \otimes \sigma$ and $e \otimes f \otimes \tau$. Assume that $m \geq n$. Then the product $d_{1} d_{2}$ either is an element of $J_{n-2}$ or is an element of $J_{n} \backslash J_{n-2}$, and in the latter case it corresponds under $\psi$ to a scalar multiple of an element $c \otimes e \otimes \mu \tau$ where $c$ is an element in $V_{n}$ and $\mu$ is an element in $k \Sigma_{n}$.

A similar assertion is valid in the case of $m \leq n$. The proof is similar to that of lemma 5.3 . 
The outcome of this lemma is that we can now define the bilinear maps $\alpha, \beta, \gamma$ which are needed for getting an iterated inflation. The precise definitions are very similar to that of $\psi$ given in Lemma 5.3 and thus we omit them.

Altogether we have proved the following theorem.

Theorem 5.6. The algebra $A=B_{k}(r, \delta)$ is an iterated inflation of group algebras of symmetric groups.

More precisely: as a free $k$-module, $A$ is equal to

$$
k \Sigma_{r} \oplus\left(V_{r-2} \otimes V_{r-2} \otimes k \Sigma_{r-2}\right) \oplus\left(V_{r-4} \otimes V_{r-4} \otimes k \Sigma_{r-4}\right) \oplus \ldots,
$$

and the iterated inflation starts with $k \Sigma_{r}$, inflates it along $V_{r-2} \otimes V_{r-2} \otimes k \Sigma_{r-2}$ and so on, ending with an inflation of $k=k \Sigma_{1}$ or $k=k \Sigma_{0}$ as bottom layer (depending on whether $r$ is odd or even).

As immediate consequences we get several results from the literature:

Corollary 5.7 (Graham and Lehrer [GL]). The Brauer algebra $B_{k}(r, \delta)$ over any commutative noetherian domain $k$ is cellular.

Corollary 5.8 (Graham and Lehrer [GL]). Let $k$ be a field. If $\delta$ is not zero, then the simple representations of the Brauer algebra $B_{k}(r, \delta)$ are indexed by pairs $(l, L(\lambda))$ where $l$ runs through the non-negative integers $r, r-2, \ldots$ and $L(\lambda)$ runs through the isomorphism classes of simple modules over $k \Sigma_{l}$.

In the case of $\delta=0$, the above assertion is also valid except that the case $l=0$ (which occurs only for $r$ even) does not contribute a simple module.

The exception in the case of $r$ even and $\delta=0$ comes from the fact that precisely then there is a piece in the iterated inflation which has $\varphi(-,-)$ equal to zero.

Corollary 5.9. (a) The radical of a Brauer algebra $B_{k}(r, \delta)$ is nilpotent of degree less than or equal to $\prod_{l} 3^{\text {nil }\left(k \Sigma_{r-2 l}\right)}$, the product of powers of 3 to the nilpotence degrees of the radicals of the group algebras occurring in the inflation.

(b) (Hanlon and Wales HW3, corollary 7.1.5) If $k$ has characteristic zero or bigger than $r$, then the radical of $B_{k}(r, \delta)$ is nilpotent of degree less than or equal to $3^{[r / 2]+1}$, where $[r / 2]$ is the integer part of $r / 2$.

Proof. An inflation of a semisimple algebra has radical of nilpotence degree up to three. Thus part (a) follows by writing a group algebra of a symmetric group as an iterated inflation. Under the assumption of (b), the occurring group algebras all are semisimple.

\section{Comparing With other CONSTRUCTIONS}

The aim of this section is to explain the differences between our construction of Brauer algebras and other constructions which are already known in the literature.

The pioneering work by Wenzl We1] constructs Brauer algebras by towers of algebras in the following sense: Fix a field $k$ and a parameter $\delta$. Then the Brauer algebra $B_{k}(r, \delta)$ contains subalgebras isomorphic to $B_{k}(r-1, \delta)$, to $B_{k}(r-2, \delta)$, and so on. The tower construction produces $B_{k}(r, \delta)$ from the smaller algebras, i.e. by an inductive procedure. If all the algebras involved are semisimple, this is a special case of Jones' basic construction.

Hanlon and Wales [HW1, HW2, HW3] went one step further and extended the tower construction to the non-semisimple case as well. In particular, they obtained a description of the radical of $B_{k}(r, \delta)$ in terms of data coming from smaller algebras. 
Our approach is different. We fix $r$ and never use any of the subalgebras $B_{k}(s, \delta)$ (for $s<r$ ), thus no induction is involved. We derive all information from the iterated inflation and the information contained therein. Of course, these filtrations have been used before, in particular in the semisimple case. Brown Brow1, Brow2. Brow3 and Hanlon and Wales (see section 2 in HW3) used the subquotients in this filtration. The new point in our approach, however, is to identify these subquotients as inflations of group algebras of symmetric groups. This does not give much new information if one restricts attention to characteristic zero (as all the references quoted before do) where these algebras are semisimple. But it is crucial in the general case where these algebras are not necessarily semisimple, but may carry highly non-trivial structures. We note that the group algebras of symmetric groups occurring in this context are not subalgebras of $B_{k}(r, \delta)$ (unless $\delta=1$ ), since the inflation procedure 'deforms' the multiplication. Therefore, no 'tower of algebras' construction could cover this situation.

Although we do not make use of the 'towers of algebras' approach, many of the results based on that are useful in our context as well. This applies, in particular, to the precise numerical results and working out of examples in HW1, HW2, HW3, which can be used in dealing with concrete examples of Brauer algebras and also for determining which values of $\delta$ are singular as defined in the next section.

\section{BLOCK DECOMPOSITION}

Throughout this section $k$ is assumed to be a field. We are going to determine the blocks of the Brauer algebra in case $\delta$ is not singular in the following sense.

Recall that a bilinear form $\varphi$ defining an inflation is called singular if $N_{\bar{\varphi}}(L, n)$ $:=\left\{\sum_{v \in V, l \in L} v \otimes v_{n} \otimes l \mid \sum_{v \in V, l \in L} \bar{\varphi}(w, v) l=0\right\}$ does not vanish for some simple $B$-module $L$ and some $n$.

We have seen that for non-singular $\varphi$, the algebra $(V \otimes V \otimes B) /(V \otimes V \otimes r a d(B))$ has a unit element (by lemma 3.5). (We note that this statement becomes wrong if one replaces the field $k$ by a noetherian domain, say a polynomial ring in one variable.) Now by proposition 4.2, the algebra $V \otimes V \otimes B$ has a unit element.

The parameter $\delta$ is called singular if for some $l$ the associated bilinear form $\varphi$ is singular. (This is well-defined since the singularity of $\varphi$ depends upon $\bar{\varphi}$ and the latter depends only on $\delta$.) As we have seen in section four, the singularity of $\delta$ is decided by the vanishing of at least one of finitely many polynomials; hence there are only finitely many singular values of $\delta$.

Based on some partial results as well as on extensive computations, Hanlon and Wales [HW1 formulated a precise conjecture (in the case of characteristic zero) for which values $\delta$ should be singular, namely only integers of 'small' absolute value. This conjecture has been proved by Wenzl We1. Thus in characteristic zero, most integer values of $\delta$ are not singular, whereas in prime characteristic it may happen (depending on the choice of $r$ ) that all 'integer' values are singular.

We will show that in the case of $\delta$ not singular the Brauer algebra $A=B_{k}(r, \delta)$ is a direct sum of the group algebras of symmetric groups occurring in the above picture of iterated inflation. Thus the blocks of $A$ are known, since the blocks of group algebras of symmetric groups are known by Nakayama's rule.

Lemma 7.1. Let $A$ be any ring and $J$ a twosided ideal in $A$. Then the inclusion $J \subset A$ induces a ring decomposition $A=J \oplus A / J$ if and only if $J$ (seen as a subalgebra of $A$ ) has a unit element. 
Proof. If $A$ has such a block decomposition, then $J$ clearly has a unit element. Conversely, assume $J$ has a unit element, say $e$. Then $J$ equals $e J e$, which is contained in $e A e$, which in turn is contained in the twosided ideal $J$ containing $e$. Thus $J$ equals $e A e$. Moreover, $\left(I d_{A}-e\right) A e$ is contained in $\left(I d_{A}-e\right) J$, which equals $\left(I d_{A}-e\right) e J$, hence it is zero. Similarly, $e A\left(I d_{A}-e\right)$ is zero. Thus $A$ is a direct sum $e A e \oplus\left(I d_{A}-e\right) A\left(I d_{A}-e\right)$ and the first summand is $J$; thus the second one is isomorphic to $A / J$.

This lemma will be used to find the block decomposition of the Brauer algebra. Alternatively, one may use the following statement, which is in terms of cellular algebras and which contains some information of independent interest.

Lemma 7.2. Let $A$ be a cellular algebra (over a field) with cell chain $0 \subset J_{1} \subset$ $\cdots \subset J_{n}=A$.

(a) If $L$ is a simple $A$-module which occurs as a composition factor of a standard module $\Delta(l)$ for some $l$, then there is some $m \geq l$ such that $J_{m} / J_{m-1}$ maps onto L. In other words: composition factors of standard modules are associated with 'higher' layers in the cell filtration.

(b) If some ideal $J_{l}$ in the cell chain has a unit element, then $A$ has a ring decomposition $A=J_{l} \oplus A / J_{l}$. If $l$ equals 1 , then in addition $J_{1}$ is a full matrix ring over $k$.

Proof. We first recall from KX1 that a cell ideal $J$ in an algebra $A$ over a field is either nilpotent (in which case its square is already zero) or generated (as a twosided ideal) by a primitive idempotent, say $f$, and in the latter case $J$ is a hereditary ideal, that is, it has the form $A f \otimes_{k} f A$ and the endomorphism ring $f A f$ equals $k$.

For the proof of (a) we use induction on $n$. If $n$ equals 1 , then $A$ is simple (see KX1], proposition 3.4), hence the assertion is obvious. Assume now that $n$ is bigger than 1 and pick any $l$. If $l$ is bigger than 1 , then both $L$ and $\Delta(l)$ are modules over the cellular algebra $A / J_{1}$, thus the assertion follows by induction. Hence we can assume $l=1$. If $J_{1}$ is nilpotent with square zero, then again both $L$ and $\Delta(l)$ are modules over the cellular quotient algebra $A / J_{1}$ and again the assertion follows by induction. In the other case, $J_{1}$ is a hereditary ideal of the form $A f \otimes f A$. In particular, the endomorphism ring $f A f$ of $\Delta(1) \simeq A f$ is isomorphic to $k$. Thus either $L$ is the unique top composition factor of $A f$ - and then it is a quotient of $J_{1}$ itself - or it is a composition factor of $\operatorname{rad}(A f)$ and hence a module over $A / J_{1}$, and again we can apply induction. This proves (a).

If the ideal $J_{l}$ has a unit element, say $e$, then $\Delta(l)$ equals $e \Delta(l)$, hence it cannot have any composition factor $L$ on which $A / J_{l}$ acts non-trivially. By part (a) also $A / J_{l}$ does not have any composition factor $L$ which occurs as a quotient of any of the ideals $J_{1}, \ldots, J_{l}$. Hence $A$ can be decomposed into $J_{l} \oplus A / J_{l}$.

In the case $l=1$ we have, moreover, that $\Delta(1)$ cannot have any other composition factors than just its top factor. Thus $J_{1}$ is a simple algebra. Since it is cellular, it must be a full matrix ring over $k$ by [KX1].

Theorem 7.3. Let $k$ be any field, $r$ any integer, and choose a parameter $\delta \in$ $k$ which is not singular for the Brauer algebra $B_{k}(r, \delta)$. Let $\Lambda$ be the set of all nonnegative integers smaller than or equal to $r$ and congruent to $r$ modulo two.

Then the Brauer algebra $B_{k}(r, \delta)$ decomposes into a direct sum

$$
B_{k}(r, \delta) \simeq \bigoplus_{l \in \Lambda} V_{l} \otimes V_{l} \otimes k \Sigma_{l}
$$


of the layers occurring when writing $B_{k}(r, \delta)$ as iterated inflation as in theorem 5.6. Moreover, $B_{k}(r, \delta)$ is Morita equivalent to

$$
\bigoplus_{l \in \Lambda} k \Sigma_{l}
$$

Hence the block decomposition of $B_{k}(r, \delta)$ is given by applying Nakayama's rule for block decomposition of group algebras of symmetric groups.

For a precise statement of Nakayama's rule we refer to [J, JK].

Proposition 4.2 implies that in the singular case the statement of the theorem is not true.

Proof. The previous section tells us how to write $B_{k}(r, \delta)$ as an iterated inflation the layers of which are inflations of group algebras of symmetric groups. Since $\delta$ is not singular, each of the layers has a unit element (by 4.2 and 3.5), hence applying 7.1 inductively we can split off layers as direct summands.

We noticed already that the theorem is not true if one replaces $k$ by a polynomial ring in one variable or if $k$ is a field, but $\delta$ is singular. The method of proof can however be used to split off some layers in the singular case as well. In case of characteristic zero, Wenzl [We1] (theorem 3.4 and corollary 3.5) obtained a block decomposition with rather different methods which seem to be restricted to this case (or rather to the case of a field $k$ over which all group algebras of symmetric groups occurring in the iterated inflation are semisimple). At present we have to leave open the following problem:

Problem. Let $k$ be a field of prime characteristic or a polynomial ring (of any characteristic). Determine the blocks of a Brauer algebra with singular parameter $\delta$.

Finally, we list a few corollaries to the theorem.

Corollary 7.4 (Brown [Brow2], Wenzl [We1] Theorem 3.2(a)). If the characteristic of $k$ is zero or bigger than $r$ and if $\delta$ is not singular, then the Brauer algebra $B_{k}(r, \delta)$ is split semisimple.

We note that under the assumptions of this corollary one may deduce branching rules and character formulae for the Brauer algebra. We do not formulate these results since they are (properly) contained in results of Wenzl [We1] and Ram Ram.

Corollary 7.5. Let $k$ be any field, $r$ any integer and $\delta$ not singular. Then a block of the Brauer algebra $B_{k}(r, \delta)$ has finite representation type, that is, there are up to isomorphism only finitely many indecomposable modules, if and only if this block is up to Morita equivalence a Brauer tree algebra. In this case, the Brauer tree is a line with no exceptional vertex.

Proof. We refer to Alp for the definition and a discussion of Brauer tree algebras and their ring structure (which is completely known). There it is proven that a block of a group algebra of a finite group (over a splitting field) has finite representation type if and only if it is a Brauer tree algebra. This applies to our situation, since any field is a splitting field for the symmetric group. In KX1 it has been shown that a Brauer tree of a cellular algebra must be a line. In [K1] it is moreover shown that in the case of an integral cellular algebra (which the Brauer algebra is) no exceptional vertex can occur. 


\section{REFERENCES}

[Alp] J.L.Alperin, Local representation theory, Cambridge University Press (1986). MR 87i:20002

[Br] R.BraueR, On algebras which are connected with the semisimple continous groups. Annals of Math. 38, 854-872 (1937).

[Brow1] W.P.Brown, Generalized matrix algebras. Canad. J. Math. 7, 188-190 (1955). MR 16:789b

[Brow2] W.P.Brown, The semisimplicity of $\omega_{f}^{n}$. Annals of Math. (2) 63, 324-335 (1956). MR 17:821g

[Brow3] W.P.Brown, An algebra related to the orthogonal group. Michigan J. Math. 3, 1-22 (1955-56). MR 17:232a

[GHJ] F.M.Goodman, P. DE la Harpe and V.F.R.Jones, Coxeter graphs and towers of algebras. MSRI Publ. 14, Springer (1989). MR 91c:46082

[GL] J.J.Graham AND G.I.Lehrer, Cellular algebras. Invent. Math. 123, 1-34 (1996). MR 97h:20016

[HW1] P.HANLON AND D.WALES, On the decomposition of Brauer's centralizer algebras. J. Alg. 121, 409-445 (1989). MR 91a:20041a

[HW2] P.HANlON and D.WALes, Eigenvalues connected with Brauer's centralizer algebras. J. Alg. 121, 446-476 (1989). MR 91a:20041b

[HW3] P.HANLON AND D.WALES, A tower construction for the radical in Brauer's centralizer algebras. J. Alg. 164, 773-830 (1994). MR 95f:20070

[J] G.JAmES, The representation theory of the symmetric groups. Springer LNM 682 (1978). MR 80g:20019

[JK] G.James AND A.Kerber, The representation theory of the symmetric group. Encyclopedia of Math. and its Appl. 16. Addison-Wesley (1981). MR 83k:20003

[Ker] S.V.Kerov, Realizations of representations of the Brauer semigroup. Zap. Nauchn. Sem. Len. (LOMI) 164, 188-193 (1987) (also: J. Soviet Math. 47, 2503-2507 (1989)). MR 89j:22029

[K1] S.KöNIG, Cyclotomic Schur algebras and blocks of cyclic defect, Canad. Math. Bull. 43, 79-86 (2000). CMP 2000:10

[KX1] S.KöNIG AND C.C.XI, On the structure of cellular algebras. In: Algebras and modules II (Geiranger 1996), CMS Conf. Proc. 24, Amer. Math. Soc., 365-386 (1998). MR 2000a:16011

[KX2] S.KÖNIG AND C.C.XI, Cellular algebras: inflations and Morita equivalences. Journal London Math. Soc. 60, 700-722 (1999). CMP 2000:11

[Ram] A.RAM, Characters of Brauer's centralizer algebras. Pacific J. Math. 169, 173-200 (1995). MR 96k:20020

[We1] H.WenzL, On the structure of Brauer's centralizer algebras. Annals of Math. 128, 173193 (1988). MR 89h:20059

[We2] H.WenzL, Quantum groups and subfactors of type $B, C$, and D. Comm. Math. Physics 133, 383-432 (1990). MR 92k:17032

Fakultät für Mathematik, Universität Bielefeld, Postfach 100131, D-33501 BieleFELD, Germany

Current address: Department of Mathematics and Computer Science, University of Leicester, University Road, Leicester, LE1 7RH, United Kingdom

E-mail address: sck5@mcs.le.ac.ak

Department of Mathematics, Beijing Normal University, 100875 Beijing, People's Republic of China

E-mail address: xicc@bnu.edu.cn 